\section{Mini-Review}

\author{
Correspondence \\ Peter Sander \\ psander@immv.unizh.ch
}

\title{
Lipoprotein synthesis in mycobacteria
}

\author{
Mandana Rezwan, ${ }^{1}$ Thomas Grau, ${ }^{1}$ Andreas Tschumi ${ }^{1}$ and Peter Sander ${ }^{1,2}$ \\ 'Institut für Medizinische Mikrobiologie, Universität Zürich, Gloriastrasse 32, CH-8006 Zürich, \\ Switzerland \\ ${ }^{2}$ Nationales Zentrum für Mykobakterien, Gloriastrasse 30, CH-8006 Zürich, Switzerland
}

\begin{abstract}
Lipoproteins are a functionally diverse class of secreted bacterial proteins characterized by an $\mathrm{N}$ terminal lipid moiety. The lipid moiety serves to anchor these proteins to the cell surface. Lipoproteins are synthesized as pre-prolipoproteins and mature by post-translational modifications. The post-translational modifications are directed by the lipobox motif located within the signal peptide. Enzymes involved in lipoprotein synthesis are essential in Gram-negative bacteria but not in Gram-positve bacteria. Inactivation of genes involved in lipoprotein synthesis attenuates a variety of Gram-positive pathogens, including Mycobacterium tuberculosis. The attenuated phenotype of these mutants indicates an important role of lipoproteins and lipoprotein synthesis in bacterial virulence. M. tuberculosis, the causative agent of tuberculosis, is one of the most devastating pathogens in the world. This article reviews recent findings on the synthesis, localization and function of lipoproteins in mycobacteria.
\end{abstract}

\section{Introduction}

Lipoproteins are a functionally heterogeneous class of proteins universally present in bacteria; typically between $1 \%$ and $3 \%$ of bacterial genomes encode lipoproteins (Babu et al., 2006; http://www.mrc-lmb.cam.ac.uk/genomes/ dolop/). Lipoproteins represent a subgroup of secreted proteins characterized by the presence of a lipobox. The lipobox motif is located in the C-terminal part of the leader peptide and consists of four amino acids [LVI/ASTVI/GAS/ C] (Babu et al., 2006). This motif functions as a recognition signal for lipid modification, which is made on the conserved and essential cysteine residue. Precursor lipoproteins are mainly translocated in a Sec-dependent manner across the plasma membrane and are modified subsequently. Recent investigations indicate that lipoproteins may also be translocated by the twin-arginine translocation (Tat) system (McDonough et al., 2005). However, this pathway presumably is of minor importance because the number of lipoproteins translocated via the Tat transporter is rather small. Modification of precursor proteins is mediated by the consecutive activity of three enzymes: phosphatidylglycerol-pre-prolipoprotein diacylglyceryl transferase (Lgt), prolipoprotein signal peptidase/signal peptidase II (LspA) and phospholipid-apolipoprotein $\mathrm{N}$ acyltransferase (Lnt) (Fig. 1). While Lgt and LspA are universally present in bacteria, Lnt has been reported to be restricted to Gram-negatives ( $\mathrm{Wu}, 1996)$. The lipid residue covalently linked to the conserved cysteine moiety is thought to allow for anchoring of proteins in biological membranes by means of hydrophobic interaction. In Grampositive bacteria, cell-associated lipoproteins are found in the plasma membrane. In Gram-negative bacteria the majority of cell-associated lipoproteins are found in the outer membrane - only about $10 \%$ stay anchored in the plasma membrane. Lipoproteins may be divided into five general groups according to their function in adhesion and invasion, cell wall synthesis, nutrient uptake, degradative processes, and sensing and transmembrane signalling (Sutcliffe \& Russell, 1995). Enzymes involved in lipoprotein synthesis (Lgt, LspA, Lnt) are essential in Gram-negative bacteria but not in Gram-positive bacteria (Wu, 1996; Leskela et al., 1999).

Mycobacteria belong to the group of GC-rich, Grampositive bacteria, although the cell envelope of these bacteria is rather complex and in some respects resembles the cell envelope of Gram-negatives (Brennan \& Nikaido, 1995). The lipid-rich outer layer in mycobacteria formed by mycolic acids and phospholipids is analogous to the outer membrane of Gram-negative bacteria. The genus Mycobacterium consists of more than 120 species (Rogall et al., 1990). The genome sequences of ten mycobacterial species have been determined or sequencing is near to completion (http://www.sanger.ac.uk; http://www.tigr.org). With respect to pathogenicity mycobacteria may be divided into obligate pathogens, opportunistic pathogens or nonpathogenic species. Mycobacterium tuberculosis and Mycobacterium leprae, the causative agents of tuberculosis and leprosy, are major human pathogens. Opportunistic infections, in particular in patients with immune suppression, may be due to a variety of mycobacteria, e.g. Mycobacterium avium-Mycobacterium intracellulare, Mycobacterium marinum, Mycobacterium kansasii and Mycobacterium chelonae. Mycobacterium smegmatis, a non-pathogenic, fast-growing mycobacterium, has been 

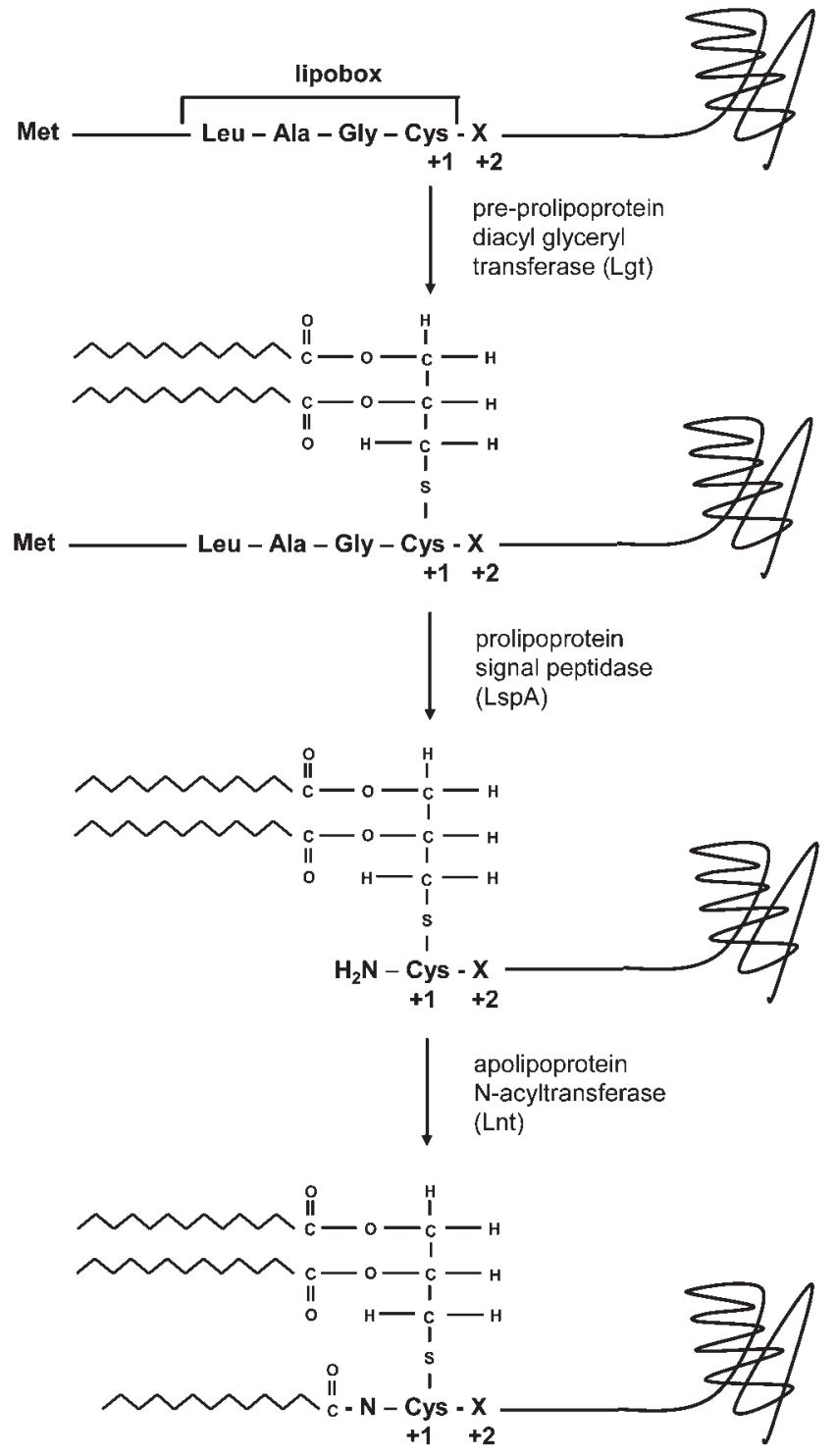

Fig. 1. Biosynthesis of bacterial lipoproteins. Precursor lipoproteins are post-translationally modified by phosphatidylglycerol-preprolipoprotein diacylglyceryl transferase (Lgt) and prolipoprotein signal peptidase (LspA). In Gram-negative bacteria and probably also in mycobacteria, lipoproteins are further processed by apolipoprotein $\mathrm{N}$-acyltransferase (Lnt).

established as a workhorse in mycobacterial genetics and biochemistry. Bioinformatic analyses suggest the presence of at least 48 lipoproteins but potentially twice as many in the genome sequence of $M$. tuberculosis (Sutcliffe \& Harrington, 2004); similar numbers are found in other mycobacteria (Table 1). Experimental data concerning mycobacterial lipoprotein function are rare. Nearly half of the annotated M. tuberculosis lipoproteins do not share conserved domains with proteins outside the genus Mycobacterium and thus represent unique proteins of mycobacteria (Sutcliffe \& Harrington, 2004). We here review recent findings on the synthesis, localization and function of lipoproteins in mycobacteria; emphasis is given to M. tuberculosis and $M$. smegmatis.

\section{Mycobacterial lipoprotein synthesis}

Lgt. Lipoproteins are synthesized as pre-prolipoproteins and mature by post-translational modifications. Lipoprotein-modifying enzymes are membrane-integral proteins located in the plasma membrane. The first step of lipoprotein synthesis is conferred by Lgt, which adds a diacylglyerol residue to the thiol group of the universally conserved cysteine within the lipobox (Fig. 1). In Escherichia coli, the lipid moiety of lipoproteins is derived from the membrane lipid phosphatidylglycerol ( $\mathrm{Wu}$, 1996). Phosphatidylglycerol is not a homogeneous entity, as its acyl chains may be composed of 16-20 carbon atoms. In addition, the acyl chains may be saturated or unsaturated. So far, lipid moieties of mycobacterial lipoproteins have not been determined at the molecular level. However, in analogy to E. coli it may be hypothesized that the lipid moieties reflect the composition of mycobacterial membrane phospholipids. Therefore, despite identical protein moieties lipoproteins may vary to some extent.

Lipid modification of proteins can be demonstrated by labelling with radioactive palmitate and by detergent solubility. Early investigations exploited incorporation of $\left[{ }^{14} \mathrm{C}\right]$ palmitic acid to demonstrate lipoprotein synthesis in M. tuberculosis (Young \& Garbe, 1991). Radioactive labelling was also used to demonstrate protein lipidation in M. smegmatis (Kriakov et al., 2003). M. tuberculosis Lgt (Rv1614) is composed of 468 aa (mol. mass $50.4 \mathrm{kDa}$ ) and thus considerably larger than its E. coli homologue (291 aa). Compared to E. coli the M. tuberculosis Lgt, as well as Lgt of other members of the order Actinomycetales, is characterized by an additional C-terminal domain of unknown function. The isoelectric points of M. tuberculosis Lgt (pI 4.45) and E. coli (pI 9.66) differ greatly. The high pI of the E. coli enzyme is assumed to be important for interaction with acidic phospholipids by ionic as well as hydrophobic interactions (Wu, 1996). The low $\mathrm{pI}$ of the M. tuberculosis Lgt enzyme is due to the unusually high aspartate and glutamate content of the C-terminal extension. In the M. smegmatis genome MSMEG3232 is annotated as Lgt. MSMEG3232 is composed of 612 aa (mol. mass $64.6 \mathrm{kDa}$ ) and like its M. tuberculosis homologue has a low $\mathrm{pI}$ (3.92). The functional consequences of the dramatic pI differences between the E. coli and the mycobacterial Lgt remain to be elucidated. In M. smegmatis a second ORF, MSMEG5388, is annotated as a putative Lgt. This ORF encodes a protein of 261 aa (mol. mass $31.0 \mathrm{kDa}$ ) with a $\mathrm{pI}$ of 9.38 . However, a multiple sequence alignment revealed that the protein encoded by MSMEG5388 differs from the Lgt consensus sequence at several conserved residues (data not shown).

LspA. Lgt-modified prolipoproteins are further processed by LspA. LspA cleaves off the signal peptide directly in front of the modified cysteine. LspA knock-out mutants therefore accumulate prolipoproteins (Fig. 1). 
Table 1. Lipoproteins in representative mycobacterial genomes

LipoP was used as a tool to predict lipoprotein signal peptides (http://www.cbs.dtu.dk/services/LipoP/; Juncker et al., 2003). Protein sequences were downloaded from the TIGR comprehensive microbial resource database (http://www.tigr.org), except for M. marinum, which was downloaded from the server of the Sanger Institute (http://www.sanger.ac.uk). The lower number of predicted lipoproteins in our search as compared to previous reports (e.g. Sutcliffe \& Harrington, 2004) is due to the more restrictive LipoP algorithm.

\begin{tabular}{|lccc|}
\hline Species & No. of predicted lipoproteins & No. of ORFs & Relative no. of lipoproteins (\%) \\
\hline M. tuberculosis & 48 & 3918 & 1.2 \\
M. leprae & 25 & 1604 & 1.6 \\
M. avium & 75 & 4350 & 1.7 \\
M. marinum & 97 & 5485 & 1.8 \\
M. smegmatis & 61 & 6776 & 0.9 \\
\hline
\end{tabular}

Due to the presence of the leader peptide, prolipoproteins have a slightly $(2-3 \mathrm{kDa})$ higher molecular mass than the mature lipoproteins. Accumulation of prolipoproteins was demonstrated in M. tuberculosis lspA (Rv1539) (Sander et al., 2004; Banaiee et al., 2006) and recently in M. smegmatis lspA (MSMEG3181) knock-out mutants (M. Rezwan, A. Tschumi, T. Grau, S. Kuhn, P. Keller, B. Springer, E. C. Böttger \& P. Sander, unpublished data). Inactivation of $M$. tuberculosis LspA by allelic replacement revealed an essential role of lipoprotein synthesis in the pathogenesis of $M$. tuberculosis. An M. tuberculosis lspA knock-out mutant exhibited reduced multiplication in the mouse macrophage cell line J774, complete absence of lung pathology and a 3-4 log reduced number of c.f.u. in a mouse model of tuberculosis infection (Sander et al., 2004). However, the molecular mechanisms underlying M. tuberculosis $l s p A$ attenuation remain to be determined.

Lipoproteins are potent agonists of TOLL-like receptor (TLR) 2, and extracts of an M. tuberculosis LspA knock-out mutant failed to induce a TLR2 response in the TLR2reporter cell line HEK293. However, TLR-dependent activation in macrophages by entire mycobacteria or whole-cell lysates was not affected by LspA inactivation (Banaiee et al., 2006), indicating the existence of TLR ligands other than mature lipoproteins. The redundancy of TLR agonists makes it unlikely that attenuation of the $\operatorname{lsp} A$ knock-out mutant is due to alterations in TLR signalling.

Globomycin, a cyclic peptide produced by several Streptomyces species, is a potent and specific inhibitor of lipoprotein signal peptidases in different bacterial species. Due to the essentiality of lipoprotein-synthesizing enzymes, globomycin exerts a bactericidal effect in E. coli (Inukai et al., 1978). Inhibition of lipoprotein maturation by globomycin, as indicated by accumulation of precursor lipoproteins, has also been shown for Gram-positive bacteria (Harrington et al., 2000). Due to the severe attenuation of the $M$. tuberculosis lspA knock-out mutant, LspA has been proposed as a putative mycobacterial drug target and exploitation of globomycin as a prototype inhibitor for further drug development has been suggested (Sander et al., 2004). Promising antibacterial activity of globomycin derivatives has recently been demonstrated in Grampositive bacteria (Kiho et al., 2004), but their activity towards mycobacteria remains to be determined.

Lnt. In Gram-negative proteobacteria, but not in Grampositive bacteria, LspA-processed lipoproteins are further modified by Lnt ( $\mathrm{Wu}, 1996)$. Lnt adds a third acyl residue to the amino group of the modified cysteine. In vitro and in vivo studies have indicated that any of the three major phospholipids in the E. coli cell envelope (phosphatidylethanolamine, phosphatidylglycerol, cardiolipin) can serve as an acyl donor ( $\mathrm{Wu}, 1996)$. In E. coli, Lnt modification is a prerequisite for transport of lipoproteins across the periplasm. Based on the assumption that $\mathrm{N}$-acylation of lipoproteins is required for transport into the mycolic acid layer, the presence of Lnt homologues in mycobacteria may be postulated. Multiple sequence alignments (data not shown) confirmed early in silico findings (Gurcha et al., 2002; Baulard et al., 2003) that Lnt homologues are present in mycobacteria. M. tuberculosis Rv2051c encodes a two-domain protein, where the N-terminal part shows similarity to E. coli Lnt. The C-terminal part of the protein encodes a polyprenolmonophosphomannose $(\mathrm{Ppm})$ synthase, which transfers mannose from GDP-mannose to endogenous polyprenol phosphates, an important metabolic intermediate in the synthesis of the mycobacterial cell wall constituents lipomannan and lipoarabinomannan (LAM). The Lnt-homology domain of Rv2051c enhances the Ppm synthase activity of the C-terminal domain, while apo-lipoprotein $\mathrm{N}$-acyltransferase activity remains to be demonstrated (Baulard et al., 2003). Besides the N-terminal domain of Rv2051c, ORF Rv2262 has lower but still significant homology to E. coli Lnt. Again, an enzymic function remains to be determined. In M. smegmatis, orthologues of the two domains of $M$. tuberculosis Rv2051c are encoded by two distinct ORFs, Msppm1 and Msppm2, of which Msppm2 (MSMEG3863) corresponds to Lnt. Thus, the M. tuberculosis Lnt ORF encodes a significantly 
larger protein (874 aa) compared to $M$. smegmatis (654 aa) and E. coli (512 aa). Lnt homologues have been found not only in mycobacteria but also in other actinobacteria, including Streptomyces and Corynebacterium. A comprehensive analysis of fully sequenced genomes of bacteria representing different phyla suggests that Lnt homologues are present in micro-organisms with a complex cell envelope (unpublished observations) but not in ordinary Gram-positive bacteria.

The presence of Lnt homologues and the localization of lipoproteins in the mycobacterial cell wall (see below) suggests that a transport system for lipoproteins into the mycolic acid layer should exist - homologous or analogous to the E. coli Lol system. The E. coli Lol system consists of (i) an ABC-transporter complex of three proteins (LolC, LolD and LolE), which releases lipoproteins from the plasma membrane; (ii) a periplasmic chaperone (LolA); and (iii) a receptor in the outer membrane (LolB), which is itself a lipoprotein. E. coli lipoproteins are translocated to the outer membrane unless they possess a Lol-avoidance signal, i.e. an aspartate at position +2 (Narita et al., 2004). More complex lipoprotein transport signals may be present in other bacterial species (Schulze \& Zuckert, 2006). Protein sequence alignments so far have not identified Lol homologues in M. tuberculosis. However, recent structural analysis of $M$. tuberculosis lipoprotein LppX revealed structural homologies to E. coli LolA and LolB, although the primary substrate seems to be phthiocerol dimycocerosate rather than lipoproteins (Sulzenbacher et al., 2006).

\section{Lipoprotein localization}

The function of a protein depends on its correct localization and vice versa. In Gram-positive bacteria, cell-associated lipoproteins stay anchored in the plasma membrane. In Gram-negative bacteria, the vast majority of cell-associated lipoproteins are released from the plasma membrane and are anchored to the outer membrane (Tokuda \& Matsuyama, 2004). Despite recent progress in subcellular fractionation of mycobacteria (Mawuenyega et al., 2005; Rezwan et al., 2006), localization of mycobacterial lipoproteins has rarely been addressed. M. tuberculosis LprG and M. smegmatis PhoA were shown to be anchored in the cell envelope (Kriakov et al., 2003); likewise M. tuberculosis Mpt83 was shown to be cell surface associated by electron microscopy and fluorescence cytometry (Vosloo et al., 1997; Harboe et al., 1998). Proteomic analyses of fractionated M. tuberculosis extracts identified and located 28 putative lipoproteins (Mawuenyega et al., 2005). Six lipoproteins were found in the cell wall fraction, ten in the plasma membrane fraction and seven in the cytosolic fraction; five lipoproteins were observed in two or in all three fractions. Localization of mature lipoproteins in the cytosol is questionable and these lipoproteins may represent recently synthesized (pre-pro-) proteins not yet secreted. Subcellular fractionation of $l g t, l s p A$ and $l n t$ mutants expressing recombinant proteins will help to elucidate the mechanisms underlying mycobacterial lipoprotein sorting.

\section{Lipoproteins}

In an excellent review Sutcliffe \& Harrington (2004) discussed the function of individual $M$. tuberculosis lipoproteins in great detail. We therefore limit our discussion to recent findings. Most mycobacterial lipoproteins have been predicted by a bioinformatic approach, i.e. identification of the lipobox consensus sequence within a typical signal peptide (Sutcliffe \& Harrington, 2004). Experimentally investigated lipoproteins often are immunodominant antigens. However, pleiotropic and seemingly antagonistic, i.e. pro- and anti-inflammatory effects, make it difficult to discern the mechanisms by which these proteins contribute to immunopathogenesis (Karakousis et al., 2004). Investigations with $M$. leprae $\mathrm{LpK}$ variants differing in the length of the peptide and the presence or absence of the lipid moiety, revealed that both acyl residues and peptide sequences are required for elicitation of an immune response. The inability of a non-acylated $27 \mathrm{kDa}$ antigen of $M$. tuberculosis to induce IFN- $\gamma$ secretion corroborates the importance of the lipid moiety for inducing an immune response (Hovav et al., 2004).

Targeted gene inactivation of individual lipoprotein genes, transposon site hybridization mutagenesis and vaccination studies corroborate early findings on the overall importance of lipoproteins in the immunopathogenesis of infection with M. tuberculosis. Several puative lipoproteins, e.g. LppP (Rv2330c), LprO (Rv2290), LprK (Rv0173), LpqT (Rv1016c), LpqY (Rv1235), LpqZ (Rv1244), LprG (Rv1411) and LppX (Rv2945c), are required for optimal growth in vivo (Rengarajan et al., 2005; Sassetti \& Rubin, 2003; Bigi et al., 2004). Some of these M. tuberculosis lipoproteins have homologues in mycobacteria, others represent signature proteins for the Corynebacterium, Mycobacterium, Nocardia (CMN) subgroup of the actinobacteria (Gao et al., 2006) (Table 2).

\section{Examples of mycobacterial lipoproteins}

The $19 \mathrm{kDa}$ antigen (LpqH, Rv3763) of M. tuberculosis (see Table 2 for summary of lipoproteins discussed) is a glycosylated lipoprotein. Early on, this protein was shown to induce a TLR-2-dependent bactericidal response in macrophages (Thoma-Uszynski et al., 2001). Recently, the $19 \mathrm{kDa}$ antigen was described as an adhesin, binding to the mannose receptor of THP-1 monocytic cells and thereby stimulating phagocytosis (Diaz-Silvestre et al., 2005). The $19 \mathrm{kDa}$ antigen was shown to induce interleukin- 1 and -12 , and tumour necrosis factor- $\alpha$ (TNF- $\alpha$ ) through TLR2signalling in macrophages. Prolonged exposure to the $19 \mathrm{kDa}$ lipoprotein inhibits IFN- $\gamma$ production and major histocompatibility (MHC) class II expression. These findings suggest that, at least in part, persistent TLR2 signalling enables $M$. tuberculosis to evade $\mathrm{T}$ cell responses and persist as a long-term infection. An M. tuberculosis $19 \mathrm{kDa}$ knockout mutant was reported to be slightly attenuated in IFN- $\gamma$ activated monocyte derived macrophages (Stewart et al., 2005). 
Table 2. Distribution of lipoproteins in mycobacteria and sequence identities/similarities

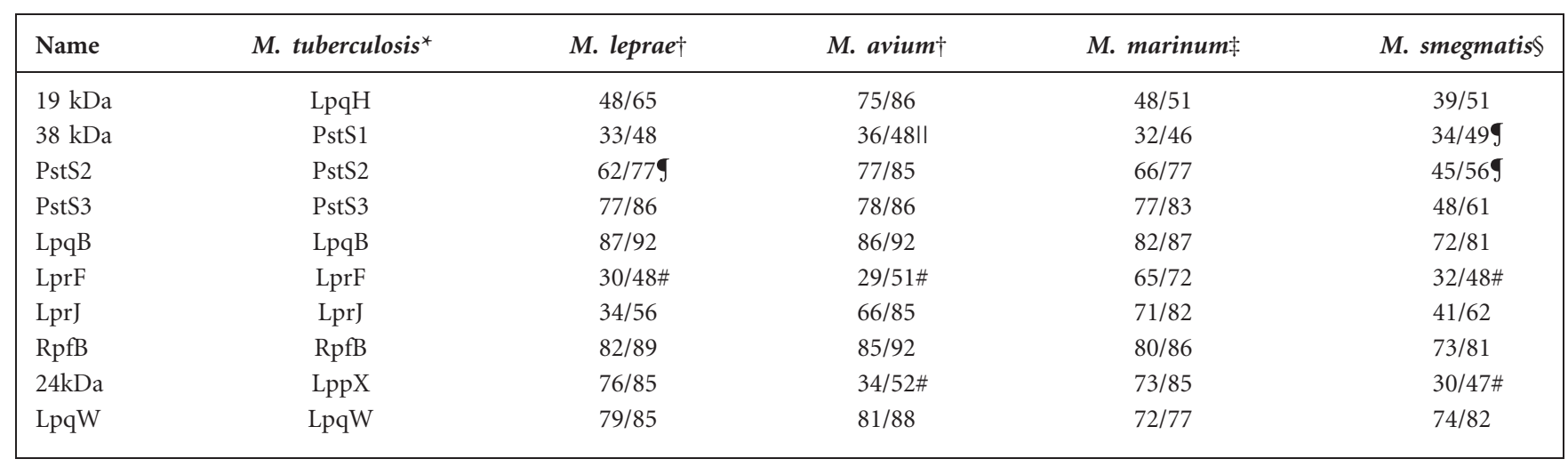

${ }^{*}$ M. tuberculosis $\mathrm{H} 37 \mathrm{Rv}$ lipoproteins were used as a query.

$\dagger$ †LAST on http://www.ncbi.nlm.nih.gov/sutils/genom_table.cgi.

‡BLAST on http://www.sanger.ac.uk/cgi-bin/blast/submitblast/m_marinum.

\$BLAST on http://tigrblast.tigr.org/cmr-blast/.

IISame ORF as identified by PstS2.

SSame ORF as identified by PstS3.

\#Same ORF as identified by LprG.

Phosphate transport receptors (Pst): three homologues of the periplasmic $\mathrm{ABC}$ phosphate-binding receptor PstS of $E$. coli have been described in M. tuberculosis [PstS1 (Rv0934), PstS2 (Rv0932c) and PstS3 (Rv0928)]. Expression of these proteins increases under phosphate-limiting conditions. $M$. tuberculosis mutants deficient in PstS1 and PstS2 showed decreased c.f.u. in lungs and spleens of mice, indicating a role in virulence (Peirs et al., 2005). Vaccination of C57BL/6 mice with PstS3 DNA protected against challenge with $M$. tuberculosis (Romano et al., 2006). Compared to PstS3 vaccination, vaccination with PstS2 and PstS1-DNA induced only modest reduction in c.f.u. counts.

$\mathrm{LpqB}(\mathrm{Rv} 3244 \mathrm{c})$ is a particularly interesting lipoprotein, as it is one of the 233 conserved signature proteins of the actinobacteria (Gao et al., 2006). lpqB is located immediately downstream of the two-component signal transduction system MtrAB. In this system MtrB is the transmembrane sensor-kinase and MtrA is the cytoplasmic response regulator. Lipoproteins have been shown to function as accessory proteins of sensor-kinase systems and it may be assumed that LpqB modulates signal sensing by MtrB (Hoskisson \& Hutchings, 2006).

Lipoproteins LprF (Rv1368) and LprJ (Rv1690) exhibited protein-protein interactions with the histidine kinase $\mathrm{KdpD}$ in a two-hybrid screen. Activation of the Kdp signal transduction pathway appears to be the primary response to environmental osmotic stress in both $M$. tuberculosis and $M$. smegmatis. The histidine kinase domain of Kdp has been suggested to form ternary complexes with LprF and LprJ and it was speculated that these proteins function as ligandbinding proteins. Co-induction of LprJ with a cluster of genes involved in cell wall integrity suggests that LprJ is involved in this process (Boshoff et al., 2004). Alternatively, LprF or LprJ could function as accessory proteins as discussed for LpqB.

RpfB (Rv1009) is the only lipoprotein among the five resuscitation-promoting factor (Rpf) proteins of $M$. tuberculosis. Rpf proteins stimulate dormant cells to divide (Keep et al., 2006). They have structural homology to glycoside hydrolases and cleave peptidoglycan; however, the exact mechanism by which these proteins promote resuscitation remains elusive. Investigations in a mouse model of $M$. tuberculosis persistence and reactivation indicated that inactivation of $r p f B$, but not inactivation of $r p f A, C, D$ or $E$, delayed reactivation, suggesting a unique role of $\mathrm{RpfB}$ in resuscitation (Tufariello et al., 2006). Whether this unique role of $\mathrm{RpfB}$ is related to the lipid anchor remains to be determined.

Investigation of LpqW and LppX revealed that these lipoproteins are key players in synthesis and transport of the unique components of the mycobacterial cell envelope. LppX ( $\mathrm{Rv2945c)}$ is a lipoprotein involved in translocation of complex lipids, the phthiocerol dimycocerosates (DIM), to the outer membrane. Structural elucidation of LppX revealed the presence of a hydrophobic cavity suitable for binding the large lipophilic side chain of DIM (Sulzenbacher et al., 2006). Orthologues of LppX are only present in mycobacteria which synthesize DIM (e.g. M. tuberculosis), and are absent from DIM-negative mycobacteria (e.g. $M$. smegmatis or M. avium). An M. tuberculosis mutant deficient in LppX is attenuated in a mouse model of infection. Attenuation is associated with a failure to release DIM into the culture supernatant rather than reduced DIM synthesis (Sulzenbacher et al., 2006). 
LpqW (Rv1166) is a highly conserved, essential lipoprotein involved in the synthesis of cell wall components. Structural analyses suggest that LpqW is derived from substrate-binding proteins, which in mycobacteria and other micro-organisms of the subfamily Corynebacterinae (also producing LAM) has evolved to match the specific needs in the synthesis of the cell wall components phosphatidyl-myo-inositol mannoside (PIM) and LAM. LpqW acts at the branching point of the PIM/LAM pathway and converts the last common intermediate, the tetramannosylated phosphatidylinositol mannoside $\mathrm{PIM}_{4}$, into the LAM pathway (Kovacevic et al., 2006; Marland et al., 2006).

\section{Conclusions}

Lipidation of proteins is required for their anchoring and sorting to the cellular surface. Recent investigations point to the importance of lipoprotein-synthesizing enzymes as well as individual lipoproteins in the biology of mycobacteria. Mycobacterial lipoproteins are crucial for synthesizing the unique mycobacterial cell envelope, sensing of and protection from environmental stress and participation in hostpathogen interaction. Due to their contribution to virulence, lipoproteins and the enzymes of the lipoprotein synthesis pathway represent promising drug targets. In addition some lipoproteins confer a protective immune response and thus may qualify as subunit vaccines. Despite progress in lipoprotein research much remains to be learned with respect to the synthesis, localization and function of mycobacterial lipoproteins and their role in host-pathogen interaction.

\section{Acknowledgements}

This work was supported by a grant from the Swiss National Science Foundation (contract 3200-068488). We thank Erik C. Böttger for continuous support and critical comments on the manuscript.

\section{References}

Babu, M. M., Priya, M. L., Selvan, A. T., Madera, M., Gough, J., Aravind, L. \& Sankaran, K. (2006). A database of bacterial lipoproteins (DOLOP) with functional assignments to predict lipoproteins. J Bacteriol 188, 2761-2773.

Banaiee, N., Kincaid, E. Z., Buchwald, U., Jacobs, W. R. \& Ernst, J. D. (2006). Potent inhbition of macrophage responses to IFN- $\gamma$ by live virulent Mycobacterium tuberculosis is independent of mature lipoproteins but dependent on TLR2. J Immunol 176, 3019-3027.

Baulard, A. R., Gurcha, S. S., Engohang-Ndong, J., Gouffi, K., Locht, C. \& Besra, G. S. (2003). In vivo interaction between the polyprenol phosphate mannose synthase Ppm1 and the integral membrane protein Ppm2 from Mycobacterium smegmatis revealed by a bacterial two-hybrid system. J Biol Chem 278, 2242-2248.

Bigi, F., Gioffre, A., Klepp, L., Santangelo, M. P., Alito, A., Caimi, K., Meikle, V., Zamarrago, M. \& other authors (2004). The knockout of the lprG-Rv1410 operon produces strong attenuation of $\mathrm{Myco}$ bacterium tuberculosis. Microbes Infect 6, 182-187.

Boshoff, H. I., Myer, T. G., Copp, B. R., McNeil, M. R., Wilson, M. A. \& Barry, C. E. (2004). The transcriptional responses of Mycobacterium tuberculosis to inhibitors of metabolism: novel insights into drug mechanisms of action. J Biol Chem 279, 40174-40184.

Brennan, P. J. \& Nikaido, H. (1995). The envelope of mycobacteria. Annu Rev Biochem 64, 29-63.

Diaz-Silvestre, H., Espinosa-Cueto, P., Sanchez-Gonzalez, A., Esparza-Ceron, M. A., Pereira-Suarez, A. L., Bernal-Fernandez, G., Espitia, C. \& Mancilla, R. (2005). The 19-kDa antigen of Mycobacterium tuberculosis is a major adhesin that binds the mannose receptor of THP-1 monocytic cells and promotes phagocytosis of mycobacteria. Microb Pathog 39, 97-107.

Gao, B., Paramanathan, R. \& Gupta, R. S. (2006). Signature proteins that are distinctive characteristics of Actinobacteria and their subgroups. Antonie van Leeuwenhoek 90, 69-91.

Gurcha, S. S., Baulard, A. R., Kremer, L., Locht, C., Moody, D. B., Muhlecker, W., Costello, C. E., Crick, D. C., Brennan, P. J. \& Besra, G. S. (2002). Ppm1, a novel polyprenol monophosphomannose synthase from Mycobacterium tuberculosis. Biochem J 365, 441-450.

Harboe, M., Wiker, H. G., Ulvund, G., Lund-Pedersen, B., Andersen, A. B., Hewinson, R. G. \& Nagai, S. (1998). Mpb70 and Mpb83 as indicators of protein localization in mycobacterial cells. Infect Immun 66, 289-296.

Harrington, D. J., Greated, J. S., Chanter, N. \& Sutcliffe, I. C. (2000). Identification of lipoprotein homologues of pneumococcal PsaA in the equine pathogens Streptococcus equi and Streptococcus zooepidemicus. Infect Immun 68, 6048-6051.

Hoskisson, P. A. \& Hutchings, M. I. (2006). MtrAB-LpqB: a conserved three-component system in actinobacteria? Trends Microbiol 14, 444-449.

Hovav, A.-H., Davidovitch, L., Nussbaum, G., Mullerad, J., Fishman, Y. \& Bercovier, H. (2004). Mitogenicity of the recombinant mycobacterial 27-kilodalton lipoprotein is not connected to its antiprotective effect. Infect Immun 72, 3383-3390.

Inukai, M., Nakajima, M., Osawa, M., Haneishi, T. \& Arai, M. (1978). Globomycin, a new peptide antibiotic with spheroblast-forming activity. Isolation and physico-chemical and biological characterization. J Antibiot 31, 421-426.

Juncker, A. S., Willenbrock, H., von Heinje, G., Nielsen, H., Brunak, S. \& Krogh, A. (2003). Prediction of lipoprotein signal peptides in Gram-negative bacteria. Protein Sci 12, 1652-1662.

Karakousis, P. C., Bishai, W. R. \& Dorman, S. E. (2004). Mycobacterium tuberculosis cell envelope lipids and the host immune response. Cell Microbiol 6, 105-116.

Keep, N. H., Ward, J. M., Cohen-Gonsaud, M. \& Henderson, B. (2006). Wake up! Peptidoglycan lysis and bacterial non-growth states. Trends Microbiol 14, 271-276.

Kiho, T., Nakayama, M., Ysuda, K., Miyakoshi, S., Inukai, M. \& Kogen, H. (2004). Structure-activity relationships of globomycin analogues as antibiotics. Bioorg Med Chem 12, 337-361.

Kovacevic, S., Anderson, D., Morita, Y. S., Patterson, J., Haites, R., McMillan, B. N. I., Coppel, R., McConville, M. J. \& Billman-Jacobe, H. (2006). Identification of a novel protein with a role in lipoarabinomannan biosynthesis in mycobacteria. J Biol Chem 281, 9011-9017.

Kriakov, J., Lee, S. H. \& Jacobs, W. R., Jr (2003). Identification of a regulated alkaline phosphatase, a cell surface-associated lipoprotein, in Mycobacterium smegmatis. J Bacteriol 185, 4983-4991.

Leskela, S., Wahlstrom, E., Kontinen, V. P. \& Sarvas, M. (1999). Lipid modification of prelipoproteins is dispensable for growth but essential for efficient protein secretion in Bacillus subtilis: characterization of the lgt gene. Mol Microbiol 31, 1075-1085.

Marland, Z., Beddoe, T., Zaker-Tabrizi, L., Lucet, I. S., Brammananth, R., Whisstock, J. C., Wilce, M. C. J., Coppel, R. L., 
Crellin, P. K. \& Rossjohn, J. (2006). Hijacking of a substrate-binding protein scaffold for use in mycobacterial cell wall biosynthesis. $J \mathrm{Mol}$ Biol 359, 983-997.

Mawuenyega, K. G., Forst, C. V., Dobos, K. M., Belisle, J. T., Chen, J., Bradbury, E. M., Bradbury, A. R. M. \& Chen, X. (2005). Mycobacterium tuberculosis functional network analysis by global subcellular protein profiling. Mol Biol Cell 16, 396-404.

McDonough, J. A., Hacker, K. E., Flores, A. R., Pavelka, M. S. \& Braunstein, M. (2005). The twin-arginine translocation pathway of Mycobacterium smegmatis is functional and required for the export of mycobacterial lactamases. J Bacteriol 187, 7667-7679.

Narita, S., Matsuyama, S. \& Tokuda, H. (2004). Lipoprotein trafficking in Escherichia coli. Arch Microbiol 182, 1-6.

Peirs, P., Lefevre, P., Boarbi, S., Wang, X. M., Denis, O., Braibant, M., Pethe, K., Locht, C., Huygen, K. \& Content, J. (2005). Mycobacterium tuberculosis with disruption in genes encoding the phosphate binding proteins PstS1 and PstS2 is deficient in phosphate uptake and demonstrates reduced in vivo virulence. Infect Immun 73, 18981902.

Rengarajan, J., Bloom, B. R. \& Rubin, E. J. (2005). Genome-wide requirements for Mycobacterium tuberculosis adaptation and survival in macrophages. Proc Natl Acad Sci U S A 102, 8327-8332.

Rezwan, M., Laneelle, M. A., Sander, P. \& Daffé, M. (2006). Breaking down the wall: fractionation of mycobacteria. J Microbiol Methods (in press).

Rogall, T., Wolters, J., Flohr, T. \& Böttger, E. C. (1990). Towards a phylogeny and definition of species at the molecular level within the genus Mycobacterium. Int J Syst Bacteriol 40, 323-330.

Romano, M., Roupie, V., Hamard, M. \& Huygen, K. (2006). Evaluation of the immunogenicity of pBudCE4.1 plasmids encoding mycolyl-transferase Ag85A and phosphate transport receptor PstS-3 from Mycobacterium tuberculosis. Vaccine 24, 3353-3364.

Sander, P., Rezwan, M., Walker, B., Rampini, S. K., Kroppenstedt, R. M., Ehlers, S. Keller, C., Keeble, J. R., Hagemeier, M. \& other authors (2004). Lipoprotein processing is required for virulence of Mycobacterium tuberculosis. Mol Microbiol 52, 1543-1552.

Sassetti, C. M. \& Rubin, E. J. (2003). Genetic requirements for mycobacterial survival during infection. Proc Natl Acad Sci U S A 100, 12989-12994.
Schulze, R. J. \& Zuckert, W. R. (2006). Borrelia burgdorferi lipoproteins are secreted to the outer surface by default. Mol Microbiol 59, 1473-1484.

Stewart, G. R., Wilkinson, K. A., Newton, S. M., Sullivan, S. M., Neyrolles, O., Wain, J. R., Patel, J., Pool, K. L., Young, D. B. \& Wilkinson, R. J. (2005). Effect of deletion or overexpression of the 19-kilodalton lipoprotein $\operatorname{Rv} 3763$ on the innate response to Mycobacterium tuberculosis. Infect Immun 73, 6831-6837.

Sulzenbacher, G., Canaan, S., Bordat, Y., Neyrolles, O., Stadthagen, G., Roig-Zamboni, V., Rauzier, J., Maurin, D., Laval, F. \& other authors (2006). LppX is a lipoprotein required for translocation of phthiocerol dimycocerosates to the surface of Mycobacterium tuberculosis. EMBO J 25, 1436-1444.

Sutcliffe, I. C. \& Harrington, D. J. (2004). Lipoproteins of Mycobacterium tuberculosis: an abundant and functionally diverse class of cell envelope components. FEMS Microbiol Rev 28, 645-659.

Sutcliffe, I. C. \& Russell, R. R. B. (1995). Lipoproteins of Grampositive bacteria. J Bacteriol 177, 1123-1128.

Thoma-Uszynski, S., Stenger, S., Takeuchi, O., Ochoa, M. T., Engele, M., Sieling, P. A., Barnes, P. F., Röllinghoff, M., Bölsckei, P. L. \& other authors (2001). Induction of direct antimicrobial activity through mammalian Toll-like receptors. Science 291, 1544-1547.

Tokuda, H. \& Matsuyama, S.-i. (2004). Sorting of lipoproteins to the outer membrane in E. coli. Biochim Biophys Acta 1693, 5-13.

Tufariello, J. A. M., Mi, K., Xu, J., Manabe, Y. C., Kesavan, A. K., Drumm, J., Tanaka, K., Jacobs, W. R. \& Chan, J. (2006). Deletion of the Mycobacterium tuberculosis resuscitation-promotion factor Rv1009 gene results in delayed reactivation from chronic tuberculosis. Infect Immun 74, 2985-2995.

Vosloo, W., Tippoo, P., Hughes, J. E., Harriman, N., Emms, M., Beatty, D. W., Zappe, H. \& Steyn, L. M. (1997). Characterisation of a lipoprotein in Mycobacterium bovis (BCG) with sequence similarity to the secreted protein MPB70. Gene 188, 123-128.

Wu, H. C. (1996). Biosynthesis of lipoproteins. In Escherichia coli and Salmonella typhimurium, pp. 1005-1014. Edited by F. C. Neidhardt and others. Washington, DC: American Society for Microbiology.

Young, D. B. \& Garbe, T. (1991). Lipoprotein antigens of Mycobacterium tuberculosis. Res Microbiol 142, 55-65. 